University of San Diego

Digital USD

Spring 5-24-2019

\title{
Reducing Hospital Readmission Rates via NP- Initiated Post Discharge Telephone Calls in Dialysis Dependent End Stage Renal Disease Patients
}

\author{
Fabiola Carapia \\ University of San Diego, carapiaf-09@sandiego.edu
}

Follow this and additional works at: https://digital.sandiego.edu/dnp

Part of the Nursing Commons

\section{Digital USD Citation}

Carapia, Fabiola, "Reducing Hospital Readmission Rates via NP- Initiated Post Discharge Telephone Calls in Dialysis Dependent End Stage Renal Disease Patients" (2019). Doctor of Nursing Practice Final Manuscripts. 104.

https://digital.sandiego.edu/dnp/104

This Doctor of Nursing Practice Final Manuscript is brought to you for free and open access by the Theses and Dissertations at Digital USD. It has been accepted for inclusion in Doctor of Nursing Practice Final Manuscripts by an authorized administrator of Digital USD. For more information, please contact digital@sandiego.edu. 


\section{Manuscript}

Reducing Hospital Readmission Rates via NP- Initiated Post Discharge Telephone Calls in Dialysis Dependent End Stage Renal Disease Patients

Fabiola Carapia, APN-BC, MSN, RN, DNP student

Kevin J. Maxwell DNP, FNP-BC, RN

Terry Behrend, MD

Hahn School of Nursing and Health Science

University of San Diego

Manuscript

April 25, 2019 


\begin{abstract}
Purpose/Aim: To determine whether a nurse practitioner-initiated phone call, in addition to the standard transition-of-care (TOC) protocol, will reduce 30-day hospital readmissions by $50 \%$ in patients with end-stage renal disease (ESRD) after an inpatient index hospitalization.
\end{abstract}

Background: $\$ 27$ billion is spent annually by Medicare for hospital readmissions. Approximately $\$ 17$ billion of this amount can be attributed to avoidable causes (Kauffman, 2016). Hospitals have been penalized for excessive readmission rates under the Hospital Readmissions Reduction Program beginning in 2012 (HRRP; Centers for Medicare \& Medicaid Services [CMS], n.d.). Index hospitalization refers to the first time, in a series of hospitalizations, that a patient is admitted for a specific condition or diagnosis. Medicare beneficiaries with ESRD, acute myocardial infarction, congestive heart failure, and pneumonia are the most common causes of readmissions.

In 2010, the Affordable Care Act (ACA) set aside $\$ 10$ billion to encourage health care providers to develop a TOC program to reduce readmission rates (Kamermayer, Leasure, \& Anderson, 2017). Several programs were developed and implemented, including HRRP and the Transition of Care Intervention Program under the ACA. In 2015, the Centers of Medicare and Medicaid Services introduced the Equity Plan for Improving Quality in Medicare (CMS, 2015). Telephone follow up calls have been shown to reduce 30-day readmission rates (Briscoe, 2018).

Process: Data from a hemodialysis clinic in southern California was /.p[;analyzed for patients with index hospitalizations. In addition to the clinic's TOC follow-up protocol, an NP-initiated phone call three days post discharge will be made for customized counseling. Phone calls will be made every two weeks. Readmission rates will be monitored weekly and at 30 days. Data will be compared to the previous 12-month readmission rate $(N=31)$ prior to the addition of the nurse practitioner phone call.

Outcomes: The goal of this project is a 50\% reduction in 30-day hospital readmissions (annual rate of 15 readmissions or less).

Conclusions: Prompt TOC with the addition of a nurse practitioner-initiated, follow-up phone call to patients with ESRD and with an index hospitalization will demonstrate a decrease in avoidable 30-day hospital readmission rates. 


\section{Introduction}

In the United States, Hospital readmissions account for billions of dollars of Medicare annual spending. Approximately \$27 billion is spent annually by Medicare for hospital readmissions. Approximately $\$ 17$ billion of this amount can be attributed to avoidable causes (Kauffman, 2016). Medicare penalizes hospitals for high readmission rates by decreasing their annual reimbursement by as much as 3\% (Barnett, Hsu, \& McWilliams, 2015). Medicare beneficiaries with acute myocardial infarction, congestive heart failure $(\mathrm{CHF})$, and pneumonia are among the most common causes of readmissions. For example, the Centers for Medicare and Medicaid Services (CMS) spends more than one-half of the cost of CHF care within this population (Cox, Lai, Lewis, \& Lenihan, 2017). CMS describes hospital readmission as admission to the hospital within thirty days of an initial hospital stay (Herbenick \& Padiernos, 2016).

Several programs have been developed and implemented, including the Hospital Readmissions Reduction Program and the Transition of Care intervention program, under the Affordable Care Act. In 2016, CMS introduced another program named Equity Plan for Improving Quality in Medicare (Betancourt, Tan-McGrory, \& Kenst, 2015).

In 2010, the Patient Protection and Affordable Care Act set aside $\$ 10$ billion to encourage health care providers to develop a transition of care program (TOC) to reduce readmission rates. TOCs act as the liaison between patients and health care settings. Initiated in the hospital setting, a TOC can include discharge instructions, post discharge appointment scheduling, and post discharge phone calls (Kamermayer, Leasure, \& Anderson, 2017). TOC intervention is an additional billing code for providers that follow up within 14 days of hospital discharge (Lapointe-Shaw, Mamdani, \& Luo, 2017). 
In October 2012, the Patient Protection and Affordable Care Act created the Hospital Readmission Reduction Program to reduced payments to hospitals with high readmission rates. This measure was designed to help organizations develop and implement evidence-based practice (EBP) interventions to reduce hospital readmissions for CMS patients with acute myocardial infarction, $\mathrm{CHF}$, and pneumonia.

In 2016, the CMS Office of Minority Health (CMS OMH) instituted a new, datadriven guideline to prevent readmissions within racially diverse Medicare beneficiaries experiencing 30-day readmissions for any medical condition. This clinical practice guideline provides action-oriented recommendations for patients with multiple chronic medical conditions. Also included is a 7-step recommendation for hospital leaders to follow for the prevention of hospital readmissions. Examples of initiatives and strategies known to reduce readmissions in ethnically diverse populations are provided as well (Betancourt et al., 2015).

Based on CMS data for minority CHF patients, African Americans had the highest incidence of CHF (4.6 per 1000 persons), followed by Hispanics (3.5 per 1000 persons) and Chinese Americans (1.0 per 1000 persons). African American and Hispanic patients had disproportionately high rates of hospital readmissions when compared with non-Hispanic Caucasians (Betancourt et al., 2015).

End stage renal disease (ESRD) patients receiving hemodialysis (HD) experienced a $35 \%$ readmission rate, almost double that of non-dialysis Medicare patients (Saran et al., 2017). Readmissions can affect quality of life with adverse clinical results. 


\section{Project Design}

Pender's Health Promotion Theory served as the guide for this project. Health Promotion emphasizes in preventing health problems before they occur and to reduce health care spending by the promotion of healthy life styles. This model focuses on positive motivation through behavioral counseling to promote higher levels of wellbeing. Pender's model emphasized three major factors, behavioral and situational factors, interpersonal influences, and biological and biographic aspects (Galloway, 2003).

The Pender model is a strong archetype for this project because it focuses on health promotion to reduce health care costs. Hemodialysis patients received a customized motivational phone call to identify their risks factors avoid readmission to the hospital to reduce health care costs.

The Iowa Model for implementing EBP into clinical settings is also a powerful model to promote quality of care. This model considers all aspects of the health care system (Brown, 2014). The first step in the Iowa Model is to identify a problem-focused trigger; problems that originate from financial data, risk management data, or a clinical issue. The problem identified at the study clinic is high hospital re-admission in ESRD Medicare beneficiaries receiving HD. The purpose of this project was to decrease the readmission of ESRD patients on HD to $9 \%$ by January 2019. The reduction of 30-day readmission for this group of patients is a priority for this practice. Team members included a pharmacist, dialysis registered nurse, registered dietitian, and the medical director. A review and analysis of the EBP literature was used to support changes. Implementing those changes and monitoring outcomes is the last step of the model (Brown, 2014). 


\section{Evidence Based Intervention}

This EBP project evaluated the effectiveness of a nurse practitioner-initiated phone call in addition to the standard of transition of care protocol to reduce hospital readmissions in dialysis dependent patients with end stage renal disease after an inpatient index hospitalization. The TOC visit included a focused examination, date of admission and discharge, reason for admission, discharge diagnosis, current medications with any changes in medications, allergies, and procedures performed during the admissions, and follow up appointments. Any interruption of medications typically given in the HD clinic were noted on the TOC visit form (e.g., erythropoietin stimulating agents) as these medications are sometimes suspended during hospitalization.

A review of literature encompassed various database (e.g., PubMed, Google Scholar, CINAHL, MEDLINE). The medical subject heading (MeSH) included hospital, readmission, transition of care, 30-day readmission, ESRD, kidney disease, CMS, financial incentives, patient discharge, and follow up phone calls. The literature search generated 20 articles. Articles selected for this project were less than 10-years old. Thirteen of these articles were chosen to be utilized based on the pyramid of rating in the Melynk and Fineout-Overholt, (2015) scale of evidence.

A systematic review by Kamermayer, Leasure, \& Anderson (2017) concluded that the use of transition of care interventions such as an individualized discharge planning and phone calls after discharge will decrease avoidable readmissions. To guide the telephone call for this project, an already established TOC form was used. For this project additional counseling was given to the patients depending on their admission diagnosis. Instructions about diet and fluid recommendation was added to the telephone guide form. 
During the TOC visit, verbal and written information was given to the patients; the information was customized according to the reason for the admission.

In the guide authored by Betancourt, Tan-McGrory, and Kenst (2015), TOC after discharge was a key strategy in that impacted a reduction in avoidable readmissions in diverse populations with multiple chronic conditions. The guideline also mentioned how minorities with chronic conditions were at a higher risk for readmission and included ESRD Medicare beneficiaries. A cohort study of HD patients by Flythe et al. (2016) concluded that medication evaluation and improved TOC from the hospital decreased avoidable readmissions among this patient population.

\section{Project Plan Process}

The project process and plan was submitted to Institutional Review Board (IRB) for approval before the implementation of the project. This project examined one HD clinic in the South Bay area of San Diego County. This clinic provided HD treatment to approximately 200 ESRD patients from diverse backgrounds and ranging in age from 40 to 80 years with multiple chronic conditions. Within that population, 80 patients were selected; patients with an index hospitalization. Once a hospital discharge summary containing important information about the patient's hospitalization was sent to the outpatient HD clinic, a face-to-face TOC occurred after the patient's first visit for HD treatment post hospital discharge. The TOC visit included a focused examination, date of admission and discharge, reason for admission, discharge diagnosis, current medications with any changes in medications, allergies, procedures performed during the admissions, and follow up appointments. Any interruption of medications, such as Mircera®, Hectorol® and Venofer®, were noted on the TOC visit form. 
Once the TOC visit was done, an NP-initiated phone call was made 3 days post hospital discharge for customized counseling. For this EBP project, patients received two phone calls per month for 3 months. These calls helped to assess the risk of readmission and review practices to optimize health considering the patients' unique demographic characteristics (e.g., age, gender) and comorbidities (e.g., diabetes mellitus; pulmonary disease; congestive heart failure; cardiovascular artery disease; peripheral vascular disease; gastrointestinal, such as gastrointestinal bleed and clostridium difficile infection, bacteremia/sepsis, other diagnosis).

These calls included education on low-potassium foods, what to eat, and which high potassium foods to avoid. Also, fluid-restriction counseling was incorporated. The medication review ensured that patients had sodium polystyrene sulfonate while they consumed a high-potassium food. All home medications were reviewed to assess patients' knowledge about current medications they might be taking as well as how medications might help them with their care. Patients were asked about follow-up appointments, if there were any issues with transportation, or other barriers to keeping these appointments. All phone calls were documented in the patient's chart and in a call $\log$, including the length of the call, summary of discussion, and plans for future followup phone calls. The phone log was reviewed weekly to assess whether goals were being achieved and to identify any barriers.

\section{Evaluation Results}

Data were collected from November 2018 through January 2019. A detailed breakdown of demographic information for index hospitalizations is displayed in Table 1 . 
Table 1

Patients with an Index Hospitalization

\begin{tabular}{lccc}
\hline & $\begin{array}{c}\text { November } \\
(n=27)\end{array}$ & $\begin{array}{c}\text { December } \\
(n=9)\end{array}$ & $\begin{array}{c}\text { January 2019 } \\
(n=11)\end{array}$ \\
\hline Age (years) & 68 & 67 & 67 \\
Gender, female (\%) & 52 & 77 & 72 \\
Hospital Diagnosis (\%) & 13 & 11 & 18 \\
$\quad$ Respiratory & 26 & 34 & 18 \\
Cardiovascular & 9 & 11 & 1 \\
Gastrointestinal & 17 & 11 & 1 \\
DM2 ESRD-related & 13 & 11 & 1 \\
Bacteremia/Sepsis & 22 & 22 & 61 \\
Other & & & \\
\hline
\end{tabular}

Patients who were readmitted to the hospital within 30 days were also analyzed.

Table 2 lists the characteristics of this group of patients. 
Table 2

Patients with 30-day readmission

\begin{tabular}{lccc}
\hline & $\begin{array}{c}\text { November } \\
(n=10)\end{array}$ & $\begin{array}{c}\text { December } \\
(n=7)\end{array}$ & $\begin{array}{c}\text { January 2019 } \\
(n=3)\end{array}$ \\
\hline Age (y) & 69 & 71 & 64 \\
Gender, female (\%) & 40 & 71 & 100 \\
Hospital Diagnosis (\%) & 10 & 0 & 33 \\
Respiratory & 20 & 42 & 0 \\
Cardiovascular & 10 & 14 & 0 \\
Gastrointestinal & 10 & 14 & 0 \\
DM2 ESRD-related & 20 & 14 & 33 \\
Bacteremia/Sepsis & 30 & 28 & 33 \\
Other & & & \\
\hline
\end{tabular}

For this clinic, in 2017 , approximately $32 \%$ of patients were admitted to the hospital. Approximately $17 \%$ of those patients were readmitted within 30 days of discharge.

\section{Discussion}

This EBP project was to determine whether a nurse practitioner-initiated phone call, in addition to the standard TOC protocol, would reduce 30-day hospital readmissions by $50 \%$ in patients with end-stage renal disease (ESRD) after an index hospitalization. Additionally, the purpose of this project was to decrease the readmission of ESRD patients on HD to $9 \%$ by January 2019 by having a prompt TOC visit completed by a nurse practitioner and a follow up phone call after the hospital discharge. Most of the patients with an index hospitalization were able to identify the risks for a 
readmission and, after the phone call, were willing to follow recommendations to avoid being re-hospitalized.

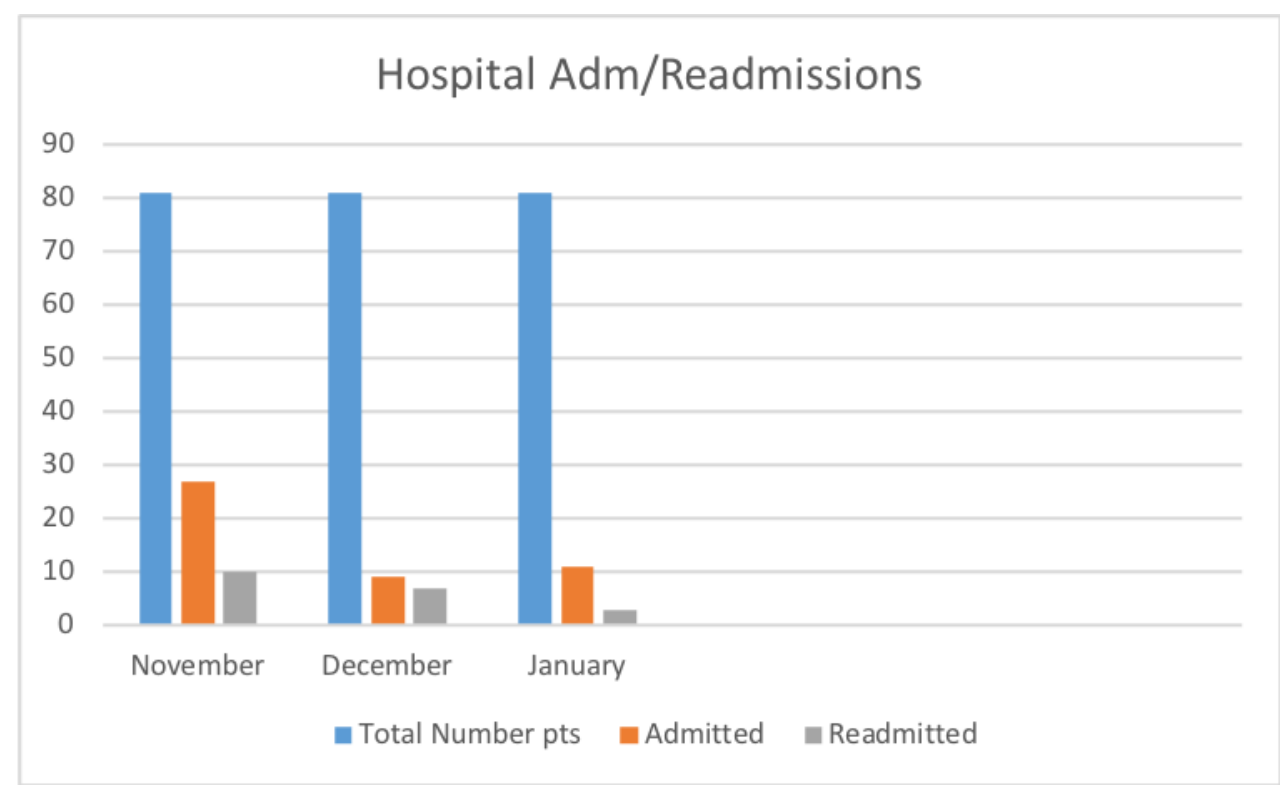

Figure 1. Percent of patients readmitted to the hospital within 30 days. 


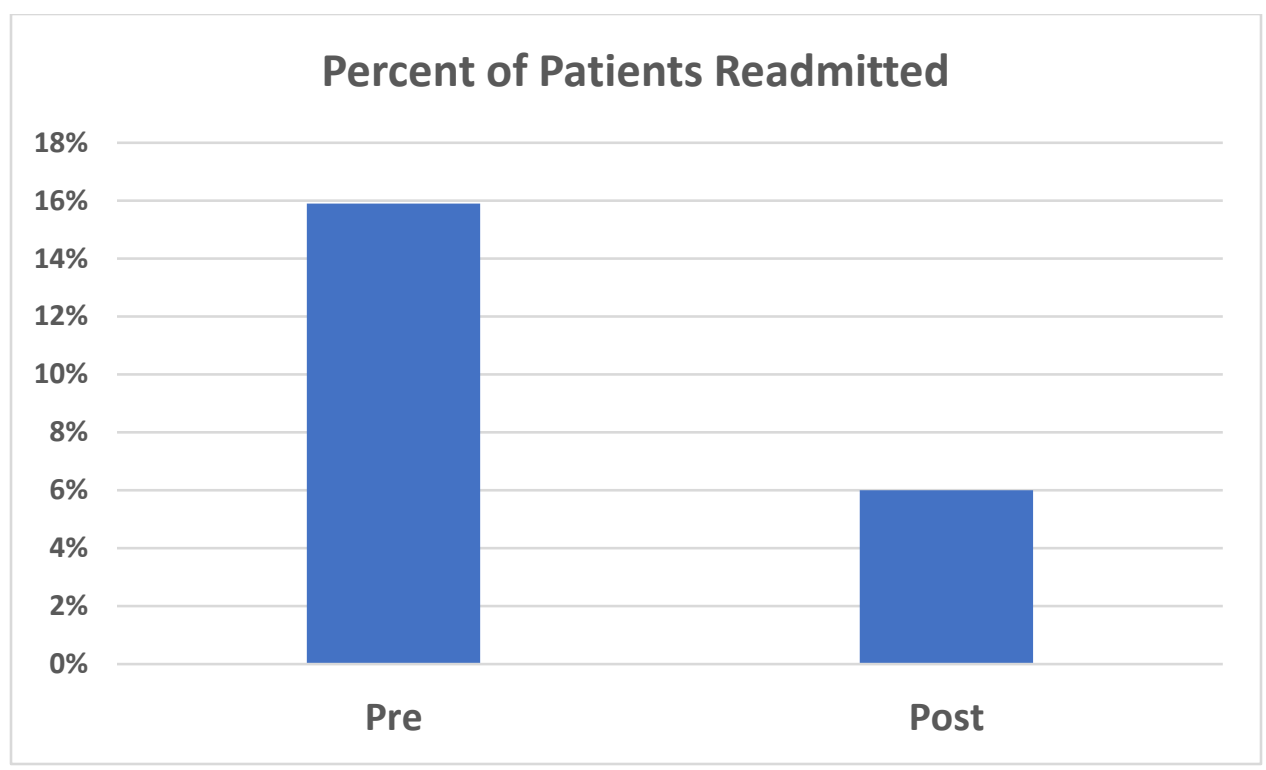

Figure 2. Percent of patients readmitted to the hospital within 30 days.

The average cost of re-admission for a patient undergoing HD is $\$ 12,000$. If a TOC visit performed on time along with educational, follow-up phone calls, readmission rates could have decreased from 31 patients to 15 patients in this practice during the study period. TOC visits increased from $80 \%$ to $98 \%$.

Table 3 summarizes the costs and cost savings after implementation of this practice. The benefit of this project included a decrease in hospital readmission from $16 \%$ $(n=31)$ to $6 \%(n=15)$ for patients with ESRD who were receiving HD. This reduction in readmissions saved the organization approximately $\$ 180,000$ in readmissions. The practice also generated more income in reimbursement revenue for every TOC visit. 
Table 3

Cost/Benefit Analysis

\begin{tabular}{|c|c|c|}
\hline & Costs & Rationale \\
\hline \multicolumn{3}{|l|}{$\underline{\text { Resources }}$} \\
\hline $\begin{array}{l}\text { TOC visit cost } \$ 250 \times 81 \\
\text { hospitalized patients }\end{array}$ & $\$ 0$ & $\begin{array}{l}\text { Reimbursement included as part of } \\
\text { bundled payments. }\end{array}$ \\
\hline $\begin{array}{l}15 \text { min telephone call x } 81 \\
\text { patients }\end{array}$ & $\$ 0$ & $\begin{array}{l}\text { No cost as performed by salaried staff } \\
\text { and completed during normal } \\
\text { working hours. }\end{array}$ \\
\hline $\begin{array}{l}\text { Call guide documents } \\
100 \text { copies X } \$ 0.10 \text { each }\end{array}$ & $\$ 10$ & $\begin{array}{l}\text { Document guide to assist patients } \\
\text { during phone call follow-ups }\end{array}$ \\
\hline \multicolumn{3}{|l|}{ Benefits } \\
\hline $\begin{array}{l}\text { Readmission cost per patient } \\
\quad \$ 12,000 \times 31\end{array}$ & $\$ 372,000$ & Average readmission cost \\
\hline $\begin{array}{l}\text { Readmission cost per patient } \\
\quad \$ 12,000 \times 15\end{array}$ & $\$ 180,000$ & Reimbursement \\
\hline$\frac{\text { Cost Savings }}{\text { Avoided patient readmits x } 16}$ & $\$ 192,000$ & $\begin{array}{l}\text { Cost avoidance from prevented } \\
\text { readmissions }\end{array}$ \\
\hline
\end{tabular}

To accomplish a $50 \%$ reduction in 30 -day hospital readmissions, from $15 \%$ to $9 \%$, clinical data were analyzed on patients meeting the above criteria. Additionally, the nurse practitioner contacted specific patients in accordance to their TOC, then monitored outcomes weekly and at 1 month. Data were compared with those patients who did not receive this nurse-practitioner intervention. Results directed the needed elements to implement change into practice.

\section{Implications for Clinical Practice and Sustainability}

Patients often visit the dialysis facility 1- to 2-days after hospital discharge;

therefore, providers rounding at hemodialysis clinics were an important factor to lowering the re-admission rate in dialysis patients. With a prompt face-to-face TOC visit, 
increased visit time, and a follow up phone call, data would demonstrate a reduction hospital readmission rates.

To ensure sustainability of this EBP project intervention, dissemination of the EBP project findings were presented at a stakeholders' meeting on March 5, 2018. To ensure that this project created a lasting impact, it was imperative to consider elements and measures in which sustainability could be preserved. Having the templates to collect data for the follow-up phone call was available to the providers in the EHR, generated remainders and automatically set follow up phone calls.

\section{Conclusion}

In the United States, hospital readmissions are responsible for billions of dollars of Medicare spending annually. Approximately $\$ 27$ billion is spent each year by Medicare for hospital readmissions. Approximately $\$ 17$ billion of this amount can be attributed to avoidable causes (Kauffman, 2016). Efforts to reduce healthcare spending has become a priority for the health care leaders and policymakers (Harel, Wald, McArthur, Chertow, \& Bell, 2015) through the programs, initiatives, and incentives that were introduced. ESRD patients receiving hemodialysis (HD) experience a readmission rate almost twice that of non-dialysis Medicare patients (Saran et al., 2017).

Readmissions can affect quality of life with adverse clinical results. EBP methods, particularly prompt TOC and an additional NP-initiated phone call, provided evidence for a reduction in hospital readmission rates.

Hospital readmissions were higher among racially- and ethnically-diverse Medicare and MediCal beneficiaries, or older than 65 years old, and with multiple chronic conditions. This hospital-readmissions project reduced preventable readmissions 
to the hospital and therefore prevented adverse clinical outcomes, such as in -hospital

medical errors, infections, hemoglobin- and albumin drops. The main goal of this project was the promote patients' quality of life in a cost-effective manner. 


\section{References}

Barnett, M. L., Hsu, J., \& McWilliams, J. M. (2015). Patient characteristics and differences in hospital readmission rates. JAMA Internal Medicine, 175, 18031812. doi:10.1001/jamainternmed.2015.4660

Betancourt, J. R., Tan-McGrory, A., \& Kenst, K. S. (2015). Guide to preventing readmissions among racially and ethnically diverse Medicare beneficiaries. Retrieved from https://eldercarebroker.com/pub/OMH_Readmissions_Guide.pdf

Brown, C. G. (2014). The Iowa Model of Evidence-Based Practice to Promote Quality Care: An Illustrated Example in Oncology Nursing. Clinical Journal Of Oncology Nursing, 18(2), 157-159. doi:10.1188/14.CJON.157-159

Briscoe, G. T. (2018). Using Post-Discharge Telephone Follow-Up by Nephrology Nurses to Reduce 30-Day Readmissions and Post-Discharge Complications for Adult Patients on Hemodialysis. Nephrology Nursing Journal, 45(3), 243-267. Retrieved from https://sandiego.idm.oclc.org/login?url=http://search.ebscohost.com/login.aspx?di rect $=$ true $\& d b=r z h \& A N=130397447 \&$ site $=$ ehost-live

Centers for Medicare \& Medicaid Services. (n.d.). Readmissions Reduction Program (HRRP). Retrieved from https://www.cms.gov/medicare/medicare-fee-forservice-payment/acuteinpatientpps/readmissions-reduction-program.html

Centers for Medicare \& Medicaid Services. (2015). The CMS Equity Plan for Improving Quality in Medicare. Retrieved from https://www.cms.gov/About-CMS/AgencyInformation/OMH/OMH_Dwnld-CMS_EquityPlanforMedicare_090615.pdf 
Cox, Z. L., Lai, P., Lewis, C. M., \& Lenihan, D. J. (2017) Centers for Medicare and Medicaid Services' readmission reports inaccurately describe an institution's decompensated heart failure admissions. Clinical Cardiology, 40, 620-625. doi:10.1002/clc.22711

Flythe, J. E., Katsanos, S. L., Hu, Y., Kshirsagar, A. V., Falk, R. J., \& Moore, C. R. (2016). Predictors of 30-Day Hospital Readmission among Maintenance Hemodialysis Patients: A Hospital's Perspective. Clinical Journal of the American Society of Nephrology, 11, 1005-1014. doi.org/10.2215/CJN.11611115 Galloway, D.R., (2003). Heath promotion, beliefs and measurements. Clinical medicine and Research, 249-258.

Harel, Z., Wald, R., McArthur, E., Chertow, G. M., Harel, S., Gruneir, A., ... Bell, C. M. (2015). Rehospitalizations and Emergency Department Visits after Hospital Discharge in Patients Receiving Maintenance Hemodialysis. Journal of the American Society of Nephrology, 26, 3141-3150. doi:10.1681/ASN.2014060614

Herbenick, S. \& Padiernos, K. N. (2016). Hospital Readmissions. The Hospital Readmissions Program (HRRP) Under Section 3025 of the Affordable Care Act (ACA) of 2012. HP Brief for Hospital.

Kamermayer, A. K., Leasure, A. R., \& Anderson, L. (2017). The effectiveness of transitions-of-care interventions in reducing hospital readmissions and mortality: A systematic review. Dimensions of Critical Care Nursing, 36, 311-316. doi:10.1097/DCC.0000000000000266 
Kauffman, B. (2016, March 23). Readmissions \& Medicare: What's the cost? [Blog post]. Retrieved from https://www.nic.org/blog/readmissions-medicare-whats-the$\cos \mathrm{s} /$

Lapointe-Shaw, L., Mamdani, M., Jin, L., Austin, P. C., Ivers, N. M., Redelmeier, D. A., \& ... Luo, J. (2017). Effectiveness of a financial incentive to physicians for timely follow-up after hospital discharge: a population-based time series analysis.

CMAJ: Canadian Medical Association Journal, 189(39), E1224-E1229. doi:10.1503/cmaj.170092

Melnyk, B. M., \& Fineout-Overholt, E. (2014). Evidence-based practice in nursing and healthcare: A guide to best practice. Philadelphia, PA: Wolters Kluwer \& Lippincott, Williams \& Wilkins.

Saran, R., Robinson, B., Abbott, K. C., Agodoa, L. Y. C., Bhave, N., Bragg-Gresham, J., ... Shahinian, V. (2018). US Renal Data System 2017 Annual Data Report: Epidemiology of kidney disease in the United States. American Journal of Kidney Diseases, 71(Suppl. 1), A7. doi:10.1053/j.ajkd.2018.01.002 
